\title{
The Planetary Context for Life
}

\author{
TORI HOEHLER
}

NASA Ames Research Center

Working concepts of habitability in planetary science are defined by reference to the requirements and limitations of life as expressed in Earth's extant biosphere: liquid water, light or redox chemical energy, the major biogenic elements (CHNOPS) and various micronutrients, and relatively broad ranges of $\mathrm{pH}$, temperature, pressure, and ionic strength/composition. Among these, a search for liquid water has been a key driver of more than two decades of planetary exploration, and multiple bodies beyond Earth are now known or thought to host (or have hosted) extensive liquid water environments. A new wave of spacecraft observations has has potential to distinguish among these worlds with respect to the availability of energy, material resources, and clement conditions, and therefore with respect to their potential to support extant life. A comprehensive perspective on the habitability of worlds beyond Earth must ultimately also consider an environment's bearing on the potential for life to emerge, with present theories for the origin of Earth life carrying significantly different implications for the biological potential of worlds beyond Earth. This talk will survey the present understanding of liquid water-bearing environments in our solar system as it relates to both extant life and origin of life perspectives on habitability. 